\title{
The type 2 myocardial infarction following hypertension:a case report
}

rui $\mathrm{xu}^{1}$, yan zhang ${ }^{1}$, Yanping $\mathrm{Bi}^{2}$, and yan wang ${ }^{2}$

${ }^{1}$ Liaoning University of Traditional Chinese Medicine

${ }^{2}$ Jilin Hospital of Integrated Traditional Chinese and western medicine

December 22, 2021

\begin{abstract}
A 60-years-old patient with sustained chest pain was referred to hypertension. The tertiary Troponin-I concentrations,namely the biomarker of myocardial injury,were $0.19 \mathrm{ng}$ per milliliter,1.288ng per milliliterand 16.698ng per milliliter,respectively.Electrocardiogram showed ST-segment and T wave dynamic changes.Type 2 MI was confirmed.
\end{abstract}

Hosted file

\begin\{CJK\}\{UTF8\}\{gbsn\} \end\{CJK\}\selectlanguage\{english\}The type } 2 \text { myocardial infarction following hype } available at https://authorea.com/users/452208/articles/550331-the-type-2-myocardialinfarction-following-hypertension-a-case-report 